\title{
Neonatal bowel resections occurring in the NICU are associated with lower survival rates compared to the OR
}

Lauren Howser; Carly Goehring; Cassandra Anderson; Eamaan Turk; Yan Han; George Eckert; Dr. Brian Gray, MD

Section of Pediatric Surgery, Riley Hospital for Children, Department of Surgery, Indiana University School of Medicine

\section{Background and Hypothesis:}

Neonatal surgical procedures frequently occur at bedside in the neonatal intensive care unit (NICU) rather than in the operating room (OR). The goal of this study was to isolate risk factors for neonatal bowel resections occurring in the NICU, compare outcomes of bowel resections occurring both in the NICU and OR, and evaluate modifiable factors in both locations.

\section{Experimental Design or Project Methods:}

We reviewed 139 neonatal ( $<30$ days old) patients at Riley Hospital for Children who underwent bowel resection in either the NICU or the OR from 2014-2018. Statistical analysis included bivariate, multivariable, and propensity score matching analyses.

\section{Results:}

When compared to neonates who had bowel resection in the OR, the NICU group had lower mean gestational age (EGA) at birth ( 26 vs 34 weeks, $p=<0.0001$ ), lower weight at surgery (72.7\% NICU patients $<1000$ grams vs $7.6 \%$ OR patients, $p=<0.0001)$, and had the operative diagnosis of spontaneous intestinal perforation (48.8\%) or NEC (42.9\%) compared to OR patients $(18.7 \%)$ for both diagnoses $(p=<0.0001, p=0.0003)$. Bivariate analysis revealed that $E G A$ at birth (OR 0.668, $p=<0.0001$ ), APGAR 1 minute (OR 0.776, $p=0.001$ ), and pre-op respiratory $\mathrm{FiO}_{2}$ percentage (OR 2.514, $\mathrm{p}=<0.0001$ ) were significant predictors of operation location. Propensity score matching ( 15 matched pairs) showed survival rates were lower in the NICU group ( $p=0.0156$ ). Modifiable risk factors, such as the usage of a heating device during surgery $(p=<0.0001)$ and temperature monitoring during surgery $(p=<0.0001)$ were positively correlated with survival, while blood administration in the first 24 hours post-surgery $(p=0.001)$ was correlated with worse survival.

\section{Conclusions:}

There are definable characteristics of neonates who have bowel resections in the OR vs NICU, but the primary outcome difference between these groups is survival. We also identified modifiable factors that can be studied to further improve survival of patients with operations in both settings. 